\title{
Dissortative From the Outside, Assortative From the Inside: Social Structure and Behavior in the Industrial Trade Network
}

\author{
Guy Kelman \\ Racah Institute for Physics \\ Hebrew University of Jerusalem \\ Jerusalem, Israel \\ Email:superk@cs.huji.ac.il \\ David S. Brée \\ Department of Computer Science \\ University of Manchester \\ Manchester, UK \\ Email: davidSbree@gmail.com
}

\author{
Eran Manes \\ Department of Public Policy \\ Guilford Glazer Faculty of \\ Business and management \\ Ben Gurion University \\ Be'er Sheva, Israel \\ Email: msemanes@gmail.com \\ Marco Lamieri \\ Research dept. \\ Intesa SanPaolo \\ Milan, Italy \\ Email: marco.lamieri@intessanpaolo.com
}

\author{
Natasa Golo \\ Racah Institute for Physics \\ Hebrew University of Jerusalem \\ Jerusalem, Israel \\ Email: natasa.golo@gmail.com \\ Sorin Solomon \\ Racah Institute for Physics \\ Hebrew University of Jerusalem \\ Jerusalem, Israel \\ Email: sorin@huji.ac.il
}

\section{Index Terms}

networks; dissortative networks; assortative mixing; missing data; economy; industrial firms; credit rating; IOU

\section{Abstract}

It is generally accepted that neighboring nodes in financial networks are negatively assorted with respect to the correlation between their degrees. This feature would play an important 'damping' role in the market during downturns (periods of distress) since this connectivity pattern between firms lowers the chances of auto-amplifying (the propagation of) distress. In this paper we explore a trade-network of industrial firms where the nodes are suppliers or buyers, and the links are those invoices that the suppliers send out to their buyers and then go on to present to their bank for discounting. The network was collected by a large Italian bank in 2007, from their intermediation of the sales on credit made by their clients. The network also shows dissortative behavior as seen in other studies on financial networks. However, when looking at the credit rating of the firms, an important attribute internal to each node, we find that firms that trade with one another share overwhelming similarity.

We know that much data is missing from our data set. However, we can quantify the amount of missing data using information exposure, a variable that connects social structure and behavior. This variable is a ratio of the sales invoices that a supplier presents to their bank over their total sales.

Results reveal a non-trivial and robust relationship between the information exposure and credit rating of a firm, indicating the influence of the neighbors on a firm's rating. This methodology provides a new insight into how to reconstruct a network suffering from incomplete information.

\section{INTRODUCTION}

The topology of a network is the visible result of integrative processes in the underlying system. It may be possible to deduce the dynamics of the underlying system from such a network. However, the mechanism so deduced will be extremely sensitive to any data that is missing from the network. If the information on the system is incomplete the rendered network may provide a misleading picture of the system and impact our understanding of the mechanisms that created it.

Financial networks are known for being negatively assorted, i.e. neighbouring nodes in the network are dissimilar, in particular as regards the degree of their in- and out-links. Among practitioners and economists this property is desired because it renders the financial network robust to percolation (propagation of distress or growth). The knowledge that contagion rarely happens may catch us by surprise when financial shocks do indeed propagate from the local level to the national/international level. In the events preceding the 2008 financial crisis, small systemic shocks affected large proportions of the industrial and financial networks. The usual response of firms to market downturns was then amplified and the response swept across the network using the monetary (communication) channels. One reason for our lack of control over this incident was that a proportion of the communication channels that link peers were not known to the banking system: the high risk mortgages were traded in the market but the credit-unworthy clients behind them remained anonymous.

We use a network of asymmetric links to represent the exchange of goods/services for financial payment between firms (nodes); it is similar to the better known communication networks. Each link is between a supplier firm and one of its customers 
who bought a product or service from them. Our data contain a snapshot in time of many firms that provide goods and services in exchange for financial payments in the year 2007. This was the year when financial crises occurred global-wide which led to an economic downturn.

These data were collected by a single large Italian bank, fulling its function as an intermediating agent in a delayed payment procedure. The bank recorded the names of the two parties and the amounts of money that one firm, the buyer, owes the other, the supplier.

Apart from the bank, both the supplier and the buyer will have entered the face value of this contract into their own bookkeeping systems under the items accounts receivable and accounts payable.

The bank prefers to deal with suppliers rather than buyers, with large firms, rather than small, and with the credit-worthy, rather than the credit-constrained. Thus already there is a selection bias on the part of the bank.

There is, moreover, another source of bias to the information on the network related to a social element. The reader could appreciate that a firm's choice of trading partner should be strategical. First and foremost because firms buy goods that they need. Second, our data describe the outcome of a short-term relationship between suppliers and buyers: the credit, or delayed payments, mechanism. Suppliers would prefer to work with buyers that clear their debt on time. However, financially constrained suppliers will probably be willing to work with less reputable clients in order to keep their business active.

In later sections we will see that the result of combining these biases leaves a rather empty picture of the full network structure. Others working on financial trade networks have made attempts to predict the missing links. Ohnishi et. al. [1], for example, adapt a trade network by filling in at random so they could run standard flow solvers. This kind of missingness that we observe should be handled carefully, and link prediction should probably be avoided.

Before we did the analysis we expected to find (1) that there would be long production chains; (2) that firms would present all their bills of sale for discounting; so (3) there would be no difference in the ratio of bills presented/total sales between firms with different credit ratings. And finally, (4) that the credit ratings of suppliers and buyers would not be correlated.

\section{A. Dissortative networks}

Assortative mixing in networks is a term describing the correlation of 'popularity' between different nodes. Popularity is a feature attributed to a node and measured by the number of incoming links to it. A network is positively assorted if the number of incoming links to a node is positively correlated with the number of incoming links to its neighbors. In assorted networks messages can spread within a small number of steps since there are many redundant links via which a message could travel. Negatively assorted networks contain highly connected nodes that are positioned sparsely throughout the network. Thus, in this type of topology the fast spread of messages is less likely [2]. However, if attributes of nodes are known, it is possible to combine structural and behavioral information for efficient routing inside this network [3].

\section{B. The trade-credit network}

In our data, the network contains a record of financial interactions between peers. The interaction under investigation is recorded when a discount process occurs. The bare explanation of a discount on an invoice is that an owner of an invoice will sell it to a financial institute for a lower price than its face value. The buyer of the invoice will be the new creditor and will take upon himself the risk that the debtor will become insolvent. This risk is combined into the rate of discount.

Today, banks offer their customers a cheaper alternative to selling their trade bills. A customer of the bank can 'collateralize its accounts receivable': the bank, instead of buying the invoice, will extend a loan. The collateral for this loan will be the face value of the invoice. The customer of the bank is a firm that approaches the bank for a loan. Usually, when using discounted invoices, this firm will be a supplier, not a buyer. Extensive reviews of the reasons why this may be so were suggested by Omiccioli [4] and Marotta [5]. Here we note one obvious reason: the supplier needs to secure funds only for production of the goods/services, whereas the buyer needs to cover the total amount of the invoice. An invoice amount includes not only the costs of production but also the supplier's profit, a larger amount than production only. Thus, the amount by which the bank discounts is, for the supplier, part of his profits, but for the buyer it is part of his costs. Lower amounts on loan impose less risk on the lender and in return a more affordable discount is offered.

\section{Credit rating and financial costs}

In order to facilitate an efficient discounting mechanism the banks created a credit-rating procedure. When the customer of the bank requires a loan, he should qualify as credit-worthy, i.e. a borrower that is financially capable of paying back. Credit-rating is a score provided to all banks by an external entity! it is usually between 1 and 9: 1 describes the most credit-worthy and 9 describes the most credit-constrained borrower. This parameter is estimated every year from balance sheet items of the firm, and thus it is specific to the firm.

Credit-rating affects the terms on loans. When a firm believes that its bank is imposing conditions that are unreasonable, it may resort to other means of financing. In general, a firm's bank is the sole provider of loan financing and by declining a loan

\footnotetext{
${ }^{1} \mathrm{CeBi}$ - Centrale dei Bilanchi, a financial analyses service for the Italian banking system.
} 
based on its terms (interest rate) the firm must consider other financing channels, the most intuitive of which is trade-credit; the firm will ask to delay its debt to suppliers and collect immediate payments from its buyers [6]. By doing so a social component is added to the pool of financing channels, and this can be traced on the network of trading firms.

The total interest paid in one year appears in the balance sheet of the firm as 'Financial Costs'. Financial Costs normalized by Total Bank Loans corresponds with credit-rating. We will use this as a continuous proxy to the credit-rating score. The in-degree, being the number of buyers for each supplier, will be the social component, and derivations for quality of a firm's neighborhood will be combinations of degree and attributes internal to the nodes and the links (such as the sums of money that move between firms).

\section{Missing data - not at random}

In order to understand this next section we need to make a clear distinction between path length and the length of a supply chain. The path length between two nodes ' $\mathrm{o}$ ' and ' $\mathrm{d}$ ' is a network measure that counts the number of nodes needed to pass in order to reach node ' $d$ ' from node 'o'. Some nodes may be unreachable if they reside in different connected components or if the network is directional, and they reside in different strongly-linked components.

A production chain (also called a supply chain) is a directional chain of inputs. Goods pass in the 'downstream' direction and money in the 'upstream' direction. In general, a firm takes input from various suppliers and generates a single output product so the structure of a supply chain is like a tree subgraph. It is usually drawn from the wide end on the bottom (raw materials) to the root, or roots, on the top. The root level contains the firms that manufacture the end product(s). Each level of the tree corresponds to several intermediate inputs and is termed in network language 'rank'.

Since our data set is an account of the flow of money, different accounting procedures along the supply chain can cause the visible part of the chain to break apart, leaving smaller number of levels on each tree subgraph.

A computerized algorithm that traverses the paths will disregard a broken supply chain since the nodes reside in different strongly-connected components. It is expected that the algorithm will uncover longer paths between the root of the supply chain and the end product. The average length of a supply chain was estimated by Nair and Vidal [7] to be between 3.0 to 4.4 .

Since the network and the consequential dynamics that we wish to deduce are both extremely sensitive to missing link information, and since extending the network at random is likely to be a biased, given the bias already in the data due to the financial support procedure of the bank, we need to explore another method for uncovering the structure of the underlying trade network [8]. Rhodes and Jones [9] provide an interesting method for generating links using probabilistic inference (Bayesian); however, they impose strong assumptions on the priors (e.g. the proportion of links in the system that are known). Others, like Smith and Moody [10], point out that generating missing information out of a known but incomplete sample is a hard task since the different statistical measures behave with varying magnitudes across network topologies (i.e. if we were but to know what the topology really is, then a probabilistic method would help greatly to improve the sample).

Firm size is suspected to be endogenous to the bias in the sample. The reason is that the bank may try to attract large firms rather than small ones. We can expect that large firms will deal more contracts (have more buyers), have larger production facilities (requiring larger total bank loans) and greater financial costs. In order to control for firm size we normalized quantities by the firm's total bank loans or by its size, using the annual net-sales (other common options that we might have used are total salaries and number of employees).

\section{Methods}

\section{A. The data}

The two data sets at the bank which we could consult were :

- Time series of individual firm balance sheets (and Profit \& Loss statements) in the 8 years between 2002 and 2009. These data contain information that allows one to know the financial status of a firm. It will hereafter be abbreviated BS.

- Bank-mediated credit transactions of trading partners in the year 2007. Each record contains 3 fields of interest: the names the two transacting firms $(c, s)$ and the total face value of all the trade-bills (invoices) passed between each supplier-buyer pair $\left(R_{c s}\right)$, as will be defined in section III-B. This data set will be termed TC.

The data are not publicly available; they were directly accessible to only one researcher, who worked for the bank. Programs to extract summary data from these two data sources were written by us and executed on a computer which belonged to the bank. The summary data, which we possess, were then further analysed to obtain the results reported here.

In total, in BS there are balance sheets of 1.3 million firms over the 8 year-window. On average, balance sheets of 700,000 firms appear in any one year with overlap of approximately 300,000 along the timeline. In 2007 there were 703,858 firms with net-sales greater than zero (potential suppliers) and 601,535 firms had purchases greater than zero (potential buyers).

In the TC data set there are 1,578,812 firms, connected by 7,290,072 links. When intersected with the firms in BS we obtain a total of 345,403 firms connected by 2,874,830 links. 273,726 of the firms in the TC data are suppliers (have incoming links). And of the joined data set TC+BS, 140,580 are suppliers. If we remove the suppliers that are linked to buyers without BS data, 
we are left with 129,584 suppler firms, all of whom have at least one buyer with BS information. We call this set $M .122,728$ of the suppliers in $M(94 \%)$ have outgoing links and therefore are also buyers. The remaining 215,819 firms are buyers only.

An individual firm (node) assumes attributes from the BS such as firm size, credit-rating, financial costs or industrial classification. In the appendix we list the variables used as attributes.

The network is directed - arrows point in the direction of the payments. The full network looks like dandelions (many buyers per supplier). An image of the network is displayed in figure 1 .

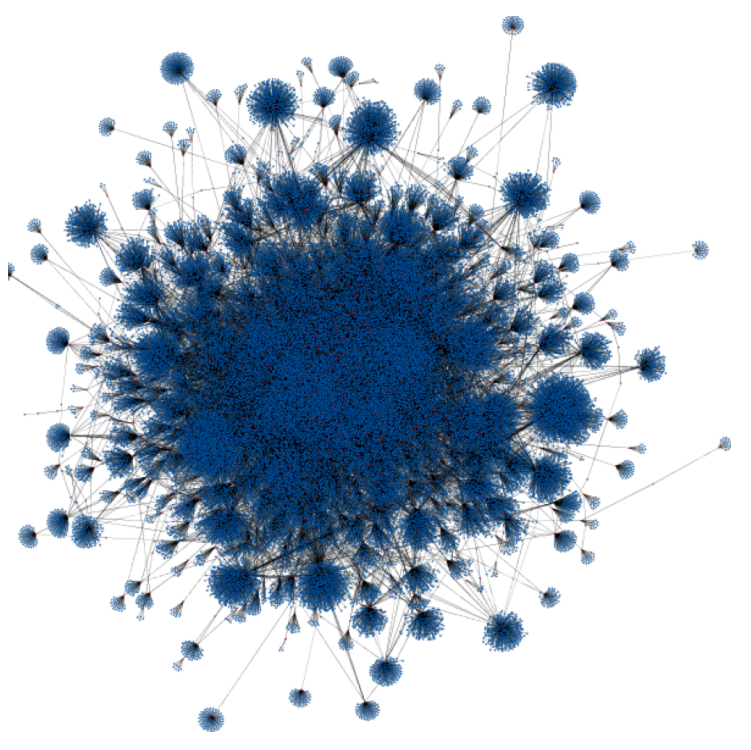

Fig. 1. Full Trade-Credit network

a) Degree distributions: The in-degree distribution forms a power law over 6 orders of magnitude (figure 2), the outdegree distribution is a curved line (can roughly be fitted with log normal), as can be expected in many kinds of networks where the generating mechanisms work on the in-degree.

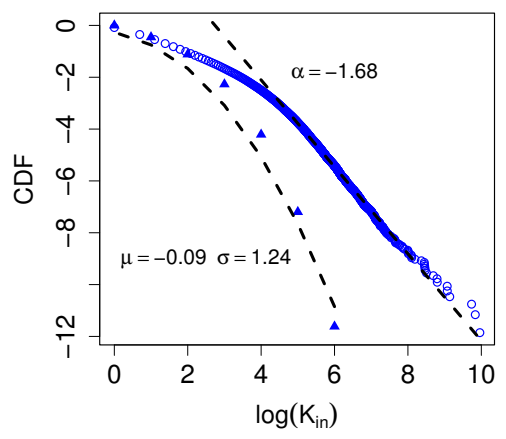

Fig. 2. Cumulative distributions of firms' in-degree (circles $N=129,584$ ) and out-degree (triangles $N=345,403$ ). The reference lines are a power law and log-normal respectively.

b) Credit Rating: A computerized system automatically assigns a RATING score to each firm with a balance sheet. The score is in the range $1 \ldots 9$ where low credit-rating is indicated by a high value. We can further group this index into classes: 'A' (RATING $=1 \ldots 3$ ) for high investment grade firms, 'B' (RATING $=4 \ldots 6)$ for speculative and 'C' $(\mathrm{RATING}=7 \ldots 9)$ that represents firms in risk of default. Firms that score into the last class are regarded by practitioners as having little or no access to bank credit. A firm with a score of 9 will rarely qualify for borrowing. However, since these firms appear in our TC data as borrowers from the bank, we assume that they did receive loans; a low RATING could be caused by the type of industry in which the firm operates.

The calculation of the RATING score is proprietary but shows correlation with Altman's Z-score [11]. A comprehensive explanation of the RATING score appears in Bottazzi et. al. [12]. 
It is, however, important to note that the RATING score of a firm is visible to all the banks with whom that firm does business. This is part of a transparent national credit system that was erected in Italy. A common credit registry is also available in other countries.

Naturally, there are more buyers than suppliers. However, the distribution of RATING scores is identical once normalized to the total number in each group. Figure 3 displays the RATING histograms of suppliers.

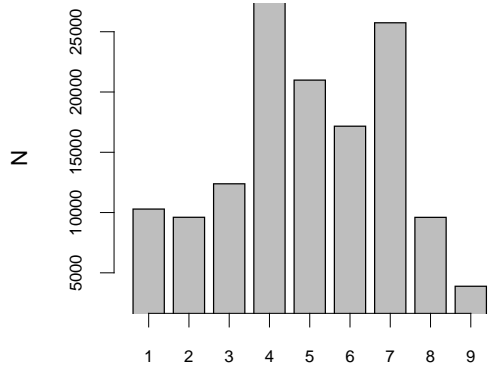

(a) frequency

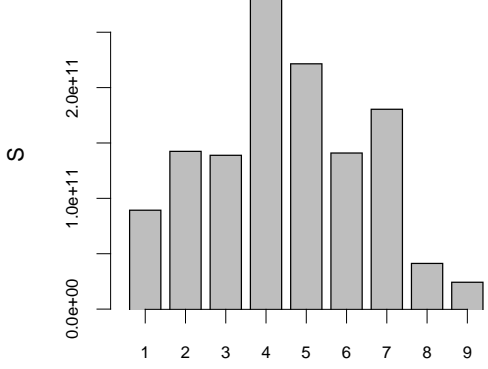

(b) size

Fig. 3. Histograms of suppliers in M: firm counts $(\mathrm{N})$ and total net-sales (S) per RATING score. RATING=1, 2,7 and 8 exhibit the largest deviation of sales per number of firms

\section{B. Definitions}

This section covers the declarations of several parameters we use in the analysis. These include the population of firms, the transaction pair, annual collateral, information exposure, and credit rating.

c) The information exposure: Let us begin by locating the quantities that affect the network visible to the bank.

We introduce here the quantity we term information exposure ' $a$ '. This is the ratio between the total amount in the invoices that supplier $s$ registered at the bank during the year, over his annual net-sales, as reported in his Profit \& Loss statement. To describe this quantity we use the following parameters.

We define the ordered pair $(c, s)$ of customer(i.e. the buyer)-supplier directional ties; each firm in the pair is a member of the population set 'Pop':

$$
(c, s) \in C S \subseteq \text { Pop } \times \text { Pop. }
$$

The amount that was presented as collateral we call $R_{c s}$. This is the annual aggregate of the invoices presented by $s$ on account of the contracts written by him to his customer $c$. The face value of an invoice serves as collateral for a short term loan in the 'credit line'. The annual total of all the invoices that $s$ presented to the bank is:

$$
R_{s}=\sum_{\{c:(c, s) \in C S\}} R_{c s}
$$

This amount should be a good proxy for the amount of the total of short term loans that $s$ received to finance production.

We can now define the information exposure, symbolized by ' $a$ '. This is the proportion of net-sales, $S_{s}$, of the supplier $s$, that he presented as collateral $R_{s}$ :

$$
a=R_{s} / S_{s}
$$

The net-sales is a parameter in the profit and loss statement of a firm and is an annual aggregate (flow) as is the enumerator $R_{s}$. ' $a$ ' is expected to be relatively close to 1 for firms running on low profit margins.

The value of ' $a$ ' is greater or equal to zero and can exceed unity. There are three possible situations:

- ' $a$ ' could be greater than one. A naive $2^{2}$ view for why $a>1$ is that there is misalignment between the time-frames in the data; the closing of the audit (P\&L statement) and the expiration of all trade-credit contracts that were signed in the same year.

- If supplier $s$ has $a=0$, the numerator in (3) vanished. The interpretation is that $s$ is not a direct client of the bank. Rather his customer, $c$, is. The customer $c$ entered $s$ into the system by executing an outgoing payment transaction.

\footnotetext{
${ }^{2}$ We assume that our data does not contain traces of illegal activity. Otherwise we would have to remove from our dataset firms that have $a>1$.
} 
- When $a \ll 1$, a discrepancy exists between the total collateral and the net-sales. This could hint that the production of $s$ requires loans that are smaller than the sales. It is an indication of a healthy use of the credit line.

In further analysis we will filter out firms having $a>2$.

Based on the introduction, the RATING score is expected to have a non-trivial relationship with information exposure. We will therefore define quantities that relate the two, such as the average information exposure for each RATING score. This is estimated over supplier firms $s$ that have rating score $r$. The set of suppliers with rating $r$ is

$$
\mathfrak{S}_{r}:=\left\{s: \mathfrak{R}_{s}=r\right\},
$$

where $\mathfrak{R}_{s}$ is the RATING score of firm $s$. The average information exposure over all firms having the same RATING is

$$
\bar{a}(r)=\frac{1}{\left|\mathfrak{S}_{r}\right|} \sum_{s \in \mathfrak{S}_{r}} a_{s},
$$

where $a_{s}$ is the information exposure parameter of supplier firm $s$.

\section{RESULTS}

\section{A. Stylized facts}

The in-degree distribution is a power law, indicating an association mechanism similar to popularity. Although the supplier is required to recruit the buyers, in the relevant literature on diffusion we witness an inevitable coupling of mass media (external factors) and word of mouth (local factors). A good historical review of market models can be found in Goldenberg et. al. [13].

Looking at other common network measures on the directed network, we find that connected component sizes are small and detached from one another with no or only links in reverse directions connecting them. The size of the giant component is 101,186 nodes with a diameter of 20 . Of the remaining nodes 239,780 are situated in small clusters of 1 link each. In many cases the central nodes are buyers with small contract sizes, e.g. phone companies or couriers, each of which is financially irrelevant to the system. Filtering out the irrelevant firms causes the network to break completely.

When counting the directional chains, the length of the longest financially relevant chain (A pays B, B pays C, C pays D, .... ) is four. However, these chains are rare and across the network there are only eight such chains. The rest of the chains are smaller. More common are chains with length of up to two.

The fact that we cannot trace the supply chain was another hint that data is severely missing.

However, there is an excellent financial explanation for this: Manufacturers are more interested in making the product, and less interested in storing and transporting it. For this task there is a dedicated industrial class called 'wholesale'. A Wholesaler will retrieve the goods from the manufacturing plant, and make sure it arrives at its destination, maybe a retailer or maybe another manufacturer down the supply chain.

The accounting procedure of wholesale is to sell the product on behalf of the manufacturer. It does not have a production cycle and acts as a goods buffer. The wholesaler delivers the goods on the condition that he gets paid for them immediately when they're sold. Consequently, it serves as a money buffer, and there is no contractual date by which the invoice must be paid. So, the bank will not accept it as collateral.

We make two assertions here: that link information is missing from the inter-firm trade network and that wholesale firms break the connection downstream along the supply chain.

\section{B. The characteristics of a supplier's neighborhood of buyers}

Generally, large firms deal with large contracts, small firms deal with small ones, although there are exceptions, e.g. the phone and courier companies. From figure 4 we learn that a large number of clients means both that the median contract size is small and with high probability the neighbor's degree is small. This is an indication that clustering around a supplier is negatively assorted. In other words, highly connected firms tend to be positioned far from one another in the network and thus render the network more vulnerable to systemic shock (removal of the highly-connected suppliers creates an impact across the whole network because their neighborhoods are not densely interconnected). However, the network is less likely to percolate in the sense that distress does not spontaneously amplify itself [2].

We created a cross tabulation of RATING scores for all suppliers in data set $M$ and their buyers that have RATING information (TC+BS), which is summarized visually in figure 5(b) The RATING of the supplier is in the columns and the RATING of the buyer in the rows. The table holds the counts of all pairs of RATING scores possible in the data. Essentially this is a description of RATING on the two ends of each link between trading firms.

The number of pairs where the supplier has RATING score greater than 8 were aggregated, leaving a total of 8 groups of supplier RATING scores. The RATING scores of buyers remain a total of 9 RATING groups.

From this table we estimated a $\chi^{2}$ test of independence of the categories. This test produced a statistic $\chi^{2}=2803$ with 56 degrees of freedom and a p-value identical to zero. The conclusion is that we can reject complete independence between 


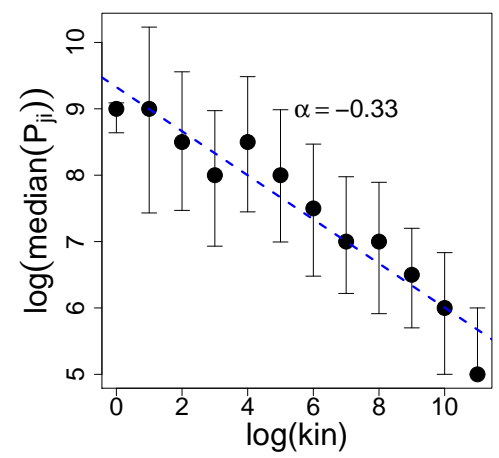

(a) mass

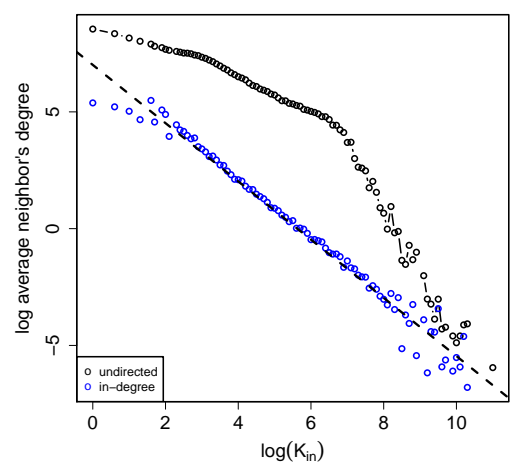

(b) number

Fig. 4. Median contract size 4(a) and average buyer (neighbor) degree 4(b) plotted against supplier's degree $\left(K\right.$ or $\left.K_{i n}\right)$. Taken from the suppliers in the trade-credit full network $(\mathrm{n}=273,726)$. The network is dissortative both in connectivity and mass. The fitting line on panel 4(b) has exponent $\alpha=-1.246$

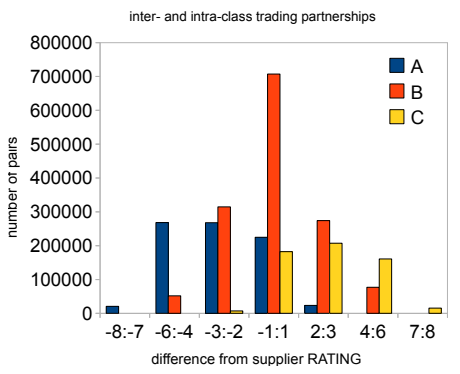

(a) difference per RATING

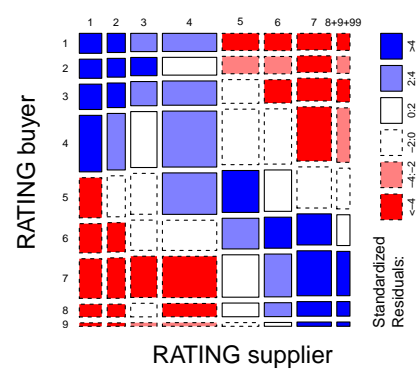

(b) mosaic

Fig. 5. Affinity between suppliers and buyers. The X-axis in panel 5(a) gives the difference supplier RATING minus buyer RATING. The Y-axis counts the number of pairs with difference categorized by $\mathrm{X}$. The suppliers are classified into 'A' ' $\mathrm{B}$ ' or ' $\mathrm{C}$ ' class, and the differences are grouped every 3. There are 2,802,976 pairs in total. Panel 5(b) shows the compressed tiles mosaic of the cross tabulation RATING supplier $\times$ RATING buyer. The plotting scheme is described by Friendly [14]

RATING of supplier and buyer and suggest a tendency of suppliers to affiliate with buyers having a similar RATING. Panel 5(b) shows the tile mosaic of the paired RATING classes with color coding that reveals this tendency; the area of each tile in the mosaic is proportional to the number of pairs where supplier has RATING=X and buyer has RATING=Y. A blue color marks significantly higher than expected occurrence, and a red color paints a significantly lower than expected pair count.

It is important to note at this stage that a $\chi^{2}$ test of independence is categorial and does not take into consideration any ordering of columns or rows. However, the table used as input maintains the original ordering in both dimensions; the RATING classes. Thus, the pattern that appears as blue along the diagonal does indicate higher-than-expected encounter of similarities in the two nodes sharing a trade-link. And any other ordering of the columns or rows would result in a less compelling pattern.

The plot in figure 5(a) shows that buyers have similar RATING scores as their suppliers. In figure 6 we see another indication that the probability of pairing with different RATING scores is decaying with the difference in RATING score. The decay is linear up to three RATING notches away, then the drop becomes sharper both in slope and in shape. This is a hint that members within the same major rating class $(1 . .3,4 . .6$ or $7 . .9)$ differentiate by the distance of RATING scores.

One reservation could be made on the result above: it is remarkable that the RATING of the supplier is so similar to the RATING score of the buyer. Looking at the sectoral affiliation of the buyers and the suppliers (figure 7) it seems that suppliers and buyers are, in the main, trading inside the same industries especially when within the manufacturing sectors (NACE categories 1 .. 3).

The visual mosaic is symmetric, i.e. it can be transposed while maintaining an almost identical pattern of red and blue tiles. One exception is NACE major category 5, wholesale, that disrupts this symmetry. Firms in industries 1 and 3 sell to firms in 5. According to our data set firms in 5 do not sell to those in 1 nor 3, but rather to those in 4 (energy). In the real world, wholesale trade does connect between manufacturers, but in our case, it only provides a transient path out of the manufacturing industries, and splits the supply chain. As explained above (III-A), this is due to the accounting procedure of wholesale and retail firms. Now, if a node is removed off the tree subgraph identified as the supply chain, it inevitably lengthens the travel 


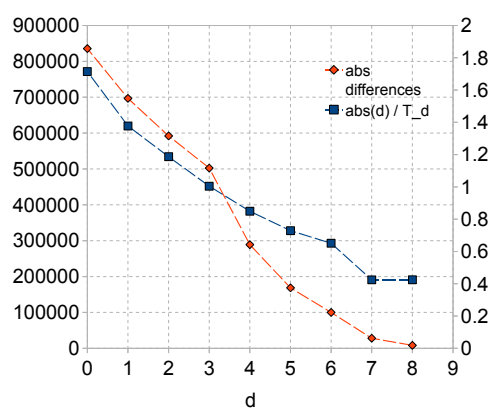

Fig. 6. Histogram of absolute RATING difference $d$ : The left Y-axis gives the number of pairs (supplier-buyer) that have an absolute difference in RATING of $d$, corresponding to the red curve. The right Y-axis is for the corrected counts (in blue). The plot shows both (1) that the number of links (between suppliers and buyers) decreases with difference $d$. (2) that the number of links (between suppliers and buyers) adjusted (for max possible) also decreases with difference.

across the network. This result supports the long path length result obtained in III-A

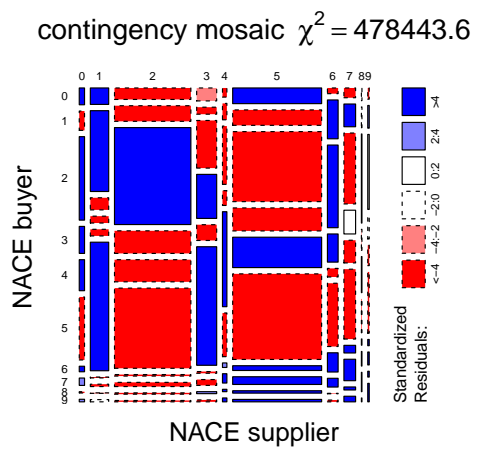

Fig. 7. Input-Output test of independence between buyer and supplier. 1-digit NACE industrial classifications are roughly: $1 \ldots 3$ manufacturing, 4 is energy, 5 is wholesale and retail, 6 is transport, and 7 is real estate.

Since sectoral affiliation is known as one of the macro-determiners of RATING scores, we addressed this conflation by creating a list of firms that trade outside their industry, and then performing the test again. Approximately 15\% of the buyersupplier links connect firms from the same 2-digit industry classification. Figure 8 shows the test of independence of RATING scores without the intra-industrial trade links. The table attached above the figure shows the sizes of the sets. The test statistic is $\chi^{2}=1456.1$ with 49 degrees of freedom and $p$-value of zero, again rejecting the hypothesis of complete independence and suggesting a trending behavior; the firms that a firm trades with have a RATING score that is similar to its own RATING score.

\begin{tabular}{|c|c|c|}
\hline classification & $\mathrm{NACE}_{i}=\mathrm{NACE}_{j}$ & $\mathrm{NACE}_{i} \neq \mathrm{NACE}_{j}$ \\
\hline 1-digit & 873,485 & $2,001,345$ \\
2-digit & 449,012 & $2,425,818$ \\
\hline
\end{tabular}

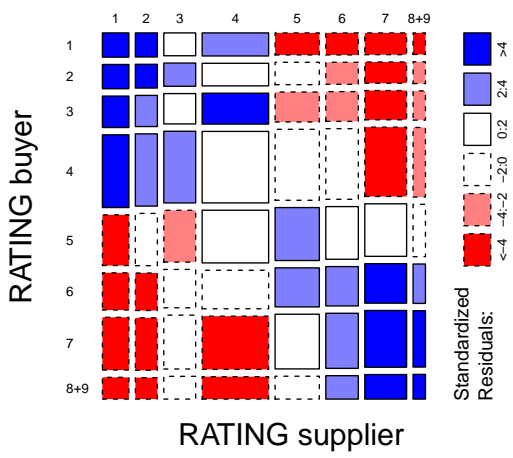

Fig. 8. The table on the top lists the number of buyer-supplier pairs from the same industry, and from other industries. NACE is the industrial classification code of the firm. On the bottom is the compressed tiles mosaic for the $\chi^{2}$ test of independence of RATING between inter-industry buyer and supplier. After removing the RATING=99 on either end there are 2,325,928 pairs. Compare to 5(b) 


\section{Analysis of the information exposure parameter}

Figure 9 shows the relation between RATING and the average information exposure, ' $a$ ', in two different forms. From this figure we see that the average information exposure is at its minimum in the middle of the RATING scale. RATING scores of firms in the 'speculative' financing group $4 \ldots 7$ have the lowest average information exposure. These firms in the middle are believed to optimize the amount of information they expose.

The distributions are skewed, so using average ' $a$ ' may not be so informative. For this reason we created a second partition of the data set. This is a division into equal-count ' $a$ '-groups: the set of 129,584 firms was ordered by ' $a$ ' and then a division into groups was made every 12,958 or 12,959 records. The lowest value of ' $a$ ' in each group is placed on the Y-axis of panel 9(b) The area of each rectangle in the mosaic is proportional to the count of sellers that have RATING=X and $a \in[Y, Y+1)$. The color code marks either significantly higher (blue) or significantly lower than expected (red) frequency of occurrence.

The effect of RATING on ' $a$ ' is a U-shape visible in both panels.

In the two extreme RATING scores, 1 and 9, the information exposure is the greatest. This sits well with the expectations that 'investment' grade firms will have dispersed their risk and therefore are indifferent to collateral quality. And that firms in risk of default will be (or think they are) forced by the bank to surrender all possible information.

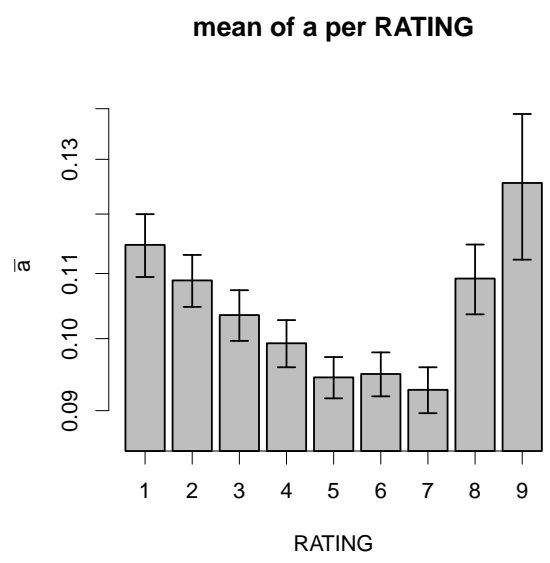

(a)

\section{contingency mosaic}

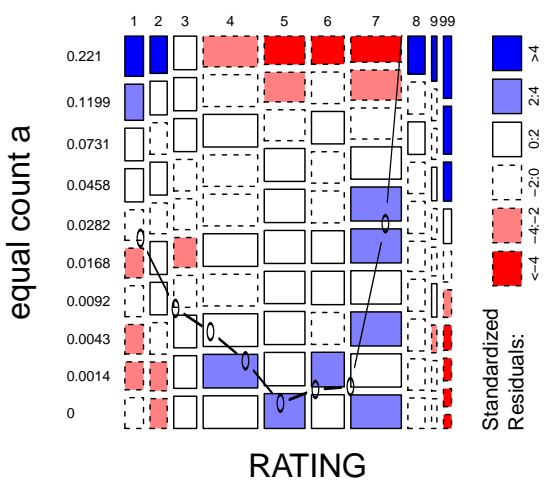

(b)

Fig. 9. RATING group $r$ vs. average information exposure parameter $\bar{a}(r)$ as defined in 5 . The bottom panel is a mosaic plot of the contingency table used for the statistical analysis. The grouping procedure is described in the text. To illustrate the U-shape there is a line plot of panel (a) stretched over panel (b)

Last, we want to show that the information exposure ' $a$ ' is correlated with the interest paid by borrowers. Figure 10 is a cross tabulation mosaic of Financial Costs (normalized by total bank loans) in the columns, and ' $a$ ' in the rows. A $\chi^{2}$ test of independence was performed and complete independence was rejected. We can observe that low information exposure is uniquely identified with borrowers that sit in the center of the interest scale. There are two more populations of borrowers that have high information exposure: firms that paid the lowest interest, and these that paid the highest.

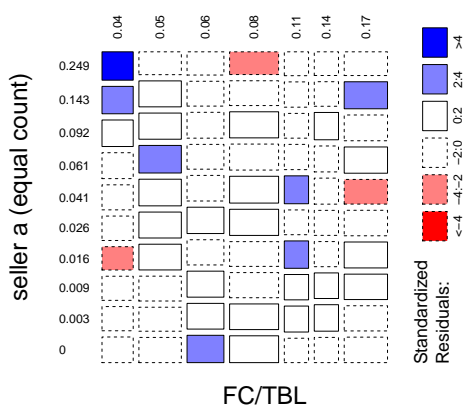

Fig. 10. Cross tabulation of the Financial Costs (interest paid) over Total Bank Loans vs. ' $a$ ' in data set $M$. The test suggests three regimes of high correlation: low information exposure firms with medium interest, and high information exposure with either high interest (low-credit rated firms) or low interest (highly credit-rated ones). The test of independence gives $\chi^{2}=142.53 d f=54$. 


\section{CONCLUSION}

The trade-network is negatively assorted in respect of degree correlations, but positively assorted when looking at the relationships between attributes of neighboring nodes. The credit-rating parameter is important to the borrower firm and the bank. In the financial sense it represents the future ability of a firm to finance its production or purchases, and implicitly it represents the ability of a node to associate with other nodes: with high probability, firms of similar rating will tend to associate. Medium credit-rated suppliers will selectively expose information about their transactions with neighbors while firms that sit on the ends of the rating scale (high or low) will be less restrictive in exposing information on their neighbors.

In the case of medium credit-rated borrowers the social component is related to an accounting behaviour on part of the borrower firm that is fondly termed in the literature 'window-dressing' [15].

In the network that we observed, a large span of data is missing. The reasons for missing information could be divided into two:

- nodes which are suppliers, are missing due to a selection bias driven by business preferences of the bank.

- nodes which are buyers and links from suppliers to existing buyers, are missing because of the interaction between the bank and the supplier (borrower).

We were able to: (a) show that data is missing but not at random and suggest that the data collector (bank) has an impact on the process by which firms tend to associate, and especially on the medium credit-rated classes; (b) show that the immediate neighborhoods of firms from the medium credit-rated classes are more likely to be missing complete links and nodes structure; (c) suggest a tendency of this network to be vulnerable to targeted removal of specific types of nodes. These types may be correlated with industries, much like the scenario of a systemic shock where the bank issues a regulatory action on the industry as a whole.

Since the bank is collecting the data and so assumes a point of view we suggest that (d) the activation of a large scale distress response due to internal dynamics is rarely visible to the bank. The synchronized response subsequent to an intervention of the regulatory system is nevertheless visible, due to the causal nature of it.

From these findings we are able to conclude for the Italian economy whenever there is a financial crisis in the banking system that:

(a) The firms with a poor RATING score will be severely hit by their bank's refusal to extended a credit line on their trade bills since they depend heavily on the bank to do so (their information exposure measure, 'a', is high.

(b) If the banks also refuse trade credit to the firms with good RATING score, they too would have to find other sources to finance their continued production; how they might do this would be pure speculation on our part.

(c) If the banks reject trade credit to middle RATING firms, these firms would not be so badly affected as they present a smaller proportion of their sales invoices for discounting.

(d) From a national perspective, it may be in the government's interest to encourage banks to continue to extend credit to the firms with poor RATING as these are the more likely to go under if their trade credit applications are refused.

Finally, the trade-network possesses a very important feature: the incentive of nodes to optimize their connectivity is measurable. To deepen our understanding of inferring structure from incomplete network information in the context of Economics, future research should be pursued in two main directions; new data and simulation:

1) We wish to locate industries that are more vulnerable to shocks. If link removal harms any industry that happens to hold most of the highly connected firms, under a dissortative regime the network could probably break up completely. It is worthwhile locating data sets of trade credit transactions in other countries. In the US, trade-bills can be traded in the market. So there are other factors that may affect the structure of that trade-network. We speculate that the US industrial trade network also appears dissortative in respect of degree correlations.

2) Simulating an assortative network is relatively easy using priors like top-cap on the firm size (see [8]). Simulating the conditions that lead a popularity-based network to visually appear dissortative may prove useful at this point.

\section{ACKNOWLEDGMENT}

We acknowledge the support of the Institute for New Economic Thinking (INET), inaugural grant number IN01100017.

\section{REFERENCES}

[1] T. Ohnishi, H. Takayasu, and M. Takayasu, "Hubs and authorities on japanese inter-firm network: Characterization of nodes in very large directed networks," Progress of Theoretical Physics Supplement, no. 79, pp. 157-166, 2009.

[2] M. E. Newman, "Assortative mixing in networks," Physical review letters, vol. 89, no. 20, p. 208701, 2002.

[3] Ö. Şimşek and D. Jensen, "Navigating networks by using homophily and degree," Proceedings of the National Academy of Sciences, vol. 105, no. 35, pp. 12 758-12 762, 09 2008. [Online]. Available: http://www.pnas.org/content/105/35/12758.abstract

[4] M. Omiccioli, "Trade credit as collateral." Temi di discussione della Banca d'Italia, Tech. Rep. 553, June 2005.

[5] G. Marotta, "Trade credit in italy: Evidence from individual firm data," Tech. Rep., 2000.

[6] M. A. Petersen and R. G. Rajan, "Trade credit: Theories and evidence," The Review of Financial Studies, vol. 10, no. 3, pp. 661-691, 10 1997. [Online]. Available: http://www.jstor.org/stable/2962200 
[7] A. Nair and J. Vidal, "Supply network topology and robustness against disruptions-an investigation using multi-agent model," International Journal of Production Research, vol. 49, no. 5, pp. 1391-1404, 2011.

[8] M. Huisman, "Imputation of missing network data: Some simple procedures," Journal of Social Structure, vol. 10, no. 1, pp. 1-29, 2009.

[9] C. J. Rhodes and P. Jones, "Inferring missing links in partially observed social networks," J Oper Res Soc, vol. 60, no. 10, pp. 1373-1383, 10 2008. [Online]. Available: http://dx.doi.org/10.1057/jors.2008.110

[10] J. A. Smith and J. Moody, "Structural effects of network sampling coverage i: Nodes missing at random," Social networks, vol. 35, no. 4, pp. 652-668, 2013.

[11] E. I. Altman, "Financial ratios, discriminant analysis and the prediction of corporate bankruptcy," The Journal of Finance, vol. 23, no. 4, pp. 589-609, 09 1968. [Online]. Available: http://www.jstor.org/stable/2978933

[12] G. Bottazzi, M. Grazzi, A. Secchi, and F. Tamagni, "Financial and economic determinants of firm default," Journal of Evolutionary Economics, vol. 21, no. 3, pp. 373-406, 2011.

[13] J. Goldenberg, B. Libai, S. Solomon, N. Jan, and D. Stauffer, "Marketing percolation," Physica A: Statistical Mechanics and its Applications, vol. 284, no. 1, pp. 335-347, 2000.

[14] M. Friendly, "Mosaic displays for multi-way contingency tables," Journal of the American Statistical Association, vol. 89, no. 425, pp. 190-200, 1994.

[15] L. Allen and A. Saunders, "Bank window dressing: Theory and evidence," Journal of Banking \& Finance, vol. 16, no. 3, pp. 585-623, 6 1992. [Online]. Available: http://www.sciencedirect.com/science/article/pii/0378426692900463

[16] E. C. eurostat, "Statistical classification of economic activities in the european community, rev. 1.1."

\section{APPENDIX}

TABLE I

NODE ATTRIBUTE NAMES AND THEIR MEANING

\begin{tabular}{|c|l|c|}
\hline name & description & source \\
\hline NACE & 1 or 2-digit industrial classification of a firm [16] & BS \\
RATING & credit-rating score $\{1,2, \ldots, 9,99\}$. & \\
& 99 is unclassified & BS \\
Size & firm net sales [EUR] & BS \\
FC & financial costs (interest paid) [EUR] & BS \\
TBL & total bank loans [EUR] & BS \\
$K_{i n}$ & number of known buyers & TC \\
$P_{j i}$ & a payment from buyer $j$ to $i$ & TC \\
$' a$ & information exposure. See equation $\sqrt{3}$ & TC,BS \\
\hline
\end{tabular}

Note: The NACE (European) industrial classification scheme is a hierarchical numbering system. The leftmost digit is the major industry code. Further resolution of sub-classifications can be achieved by adding less significant digits, up to 4 digits in the case of NACE. 